\title{
The potential impact of BCG vaccine supply shortages on global paediatric tuberculosis mortality
}

\author{
Rebecca C. Harris ${ }^{1 *}$ (D) Peter J. Dodd² and Richard G. White
}

\begin{abstract}
Background: The Bacillus Calmette-Guérin (BCG) vaccine is provided to over 100 million neonates annually to protect against childhood tuberculosis (TB). Recent BCG manufacturing interruptions highlight global supply risks. We estimated the potential impact of BCG shortfalls on global paediatric ( $<15$ years) TB mortality.

Methods: A static mathematical model was employed to estimate the number of paediatric TB deaths avoided by usual levels of BCG coverage, and potential additional TB deaths in the first 15 years of life due to 1-year BCG supply shortfalls of $6.3 \%$ (as occurred in 2015) to $27.6 \%$ (as anticipated without mitigating action in 2015) assuming no catch-up campaigns.

Results: BCG coverage without shortfalls, estimated at $90 \%$ globally, was estimated to avoid 117,132 (95 \% uncertainty range (UR): 5049-306,911) TB deaths globally per birth cohort in the first 15 years of life. An estimated 11,713 (UR: 505-30,691) additional TB deaths would occur in the first 15 years of life per $10 \%$ (26 million dose) annual supply shortfall. A 16.5 million dose (6.3 \%) shortfall as reported at the close of 2015 , reflecting $84 \%$ global coverage, was estimated as associated with 7433 (95\% UR: 320-19,477) excess TB deaths in the affected cohort in the first 15 years. A possible 24,914 (UR: 1074-65,278) additional deaths were avoided due to prompt shortfall reduction measures in 2015.

Conclusions: BCG shortages could greatly increase paediatric TB mortality. Although rapid action in 2015 minimised BCG shortfalls, avoiding a large number of potential additional deaths, the possible public health impact of even relatively small shortfalls highlights the critical importance of ensuring secure future manufacturing capacity and global BCG supply continuity.
\end{abstract}

Keywords: Bacillus Calmette-Guérin vaccine, Tuberculosis, Mycobacterium tuberculosis, Child, Paediatric, Mortality, Shortage, Shortfall, Mathematical model

\section{Background}

As one of the earliest vaccines of the World Health Organization's (WHO) Expanded Program on Immunization established in 1974, Bacillus Calmette-Guérin (BCG) is a cornerstone of childhood vaccination. It is a safe vaccine, used for neonatal vaccination in $84 \%$ of countries and provided to over 100 million neonates annually to protect against childhood tuberculosis (TB) [1].

\footnotetext{
* Correspondence: rebecca.harris@lshtm.ac.uk

'TB Modelling Group, TB Centre and Centre for the Mathematical Modelling of Infectious Diseases, Faculty of Epidemiology and Population Health, London School of Hygiene \& Tropical Medicine, Keppel Street, London WC1E 7HT, UK

Full list of author information is available at the end of the article
}

The vaccine is mostly supplied in 20-dose vials which must be used within 6 hours of opening [2,3]. Vaccine wastage is therefore common, so annual global BCG demand reaches approximately 260 million doses. Almost half of the global supply is procured through the United Nations International Children's Emergency Fund (UNICEF). Historically, these volumes have been reliably delivered [4], but sizeable shortfalls began in 2013 due to technical production difficulties. A drop in supply was experienced by both UNICEF and non-UNICEF markets, leading to several, usually self-procuring, countries approaching UNICEF for access to BCG [4]. The shortfall peaked at 22.8 million during 2014, and was 16.5 million 
at the end of $2015[5,6]$. Current measures to ensure BCG supply continuity are evidently insufficient.

It has been previously estimated that BCG averts one case of meningeal TB in the first 5 years of life per 3435 vaccinated children and one case of miliary TB for every 9314 vaccinations [7], though this may vary substantially by setting [8]. However, the impact of vaccination on TB mortality and the potential public health consequences of vaccine supply shortages have yet to be explored in the published literature. Therefore, we have used mathematical modelling to estimate the number of excess paediatric ( $<15$ years) TB deaths that could occur with various BCG shortfall scenarios.

\section{Methods}

\section{Data inputs and assumptions}

Data inputs and sources are summarised in Table 1. Our analysis assesses impact at the global level; therefore, where available, data employed are global estimates. Although manufacturing difficulties began in 2013 , shortages only began to outweigh mitigation measures in 2014 [9]; therefore, 2013 data were used as the no-shortfall baseline for model parameterisation.

The global BCG-eligible population was defined as the annual birth cohort in countries with a policy of neonatal BCG vaccination, identified as countries reporting BCG coverage to $\mathrm{WHO}$ or neonatal vaccination policy in the Global BCG Atlas [1, 10]. This was calculated from the United Nations Population Division 2010-2015 birth estimates [11], assuming equal distribution of births across this period. Although BCG is contraindicated in HIV-positive children [12], the denominator was not

Table 1 Data inputs and model assumptions

\begin{tabular}{|c|c|c|}
\hline Variable (symbol) & Point estimate (range) & Source \\
\hline \multicolumn{3}{|l|}{ Population at risk } \\
\hline $\begin{array}{l}\text { Annual global BCG-eligible } \\
\text { birth cohort }(B)\end{array}$ & 130.8 million & {$[1,10,11]$} \\
\hline Population vaccinated & HIV-negative & {$[12]$} \\
\hline $\begin{array}{l}\text { HIV-negative paediatric TB } \\
\text { deaths in } 2013 \text { (Mort) }\end{array}$ & $80,000(64,000-97,000)$ & [19] \\
\hline \multicolumn{3}{|l|}{ Vaccine deployment } \\
\hline Annual BCG demand $(D)$ & 260 million & {$[2,6]$} \\
\hline BCG coverage in 2013 (Cov) & $90 \%$ & {$[14]$} \\
\hline \multicolumn{3}{|l|}{ Vaccine characteristics } \\
\hline $\begin{array}{l}\text { Vaccine efficacy against } \\
\text { TB death (VE) }\end{array}$ & $66 \%(8-88 \%)$ & {$[16,17]$} \\
\hline Duration of protection & 15 years & {$[16]$} \\
\hline Waning of protection & None & Assumption \\
\hline Catch-up campaign & None & Assumption \\
\hline
\end{tabular}

adjusted as the proportion of the birth cohort for which BCG is contraindicated due to HIV status is negligible [13].

UNICEF report global demand of 260 million BCG doses per year $[2,6]$, with vaccination coverage estimated at $90 \%$ in 2013 [14]. BCG dose wastage at baseline $(w)$ was estimated at $54.7 \%$, in line with WHOUNICEF estimates [2], using current birth cohort $(B)$, annual BCG dose demand $(D)$, and vaccine coverage $(C o v): w=\frac{(D-(B \times C o v))}{D}$.

We explored BCG supply shortfalls of $6.3 \%, 10 \%$, $20 \%, 27.6 \%$, and $100 \%$ of the current 260 million dose global demand; $6.3 \%$ reflects the 16.5 million dose annual supply shortfall at the end of 2015. At the height of the most recent shortages, UNICEF anticipated shortfalls of up to 71.8 million doses in 2015 , equivalent to a $27.6 \%$ shortfall on usual levels; $10 \%$ and $20 \%$ are hypothetical shortfalls, of use for estimating impact of other potential scenarios. A complete stockout (100 \%) was explored to estimate the impact of each year of BCG vaccination. It was assumed that there would be no catch-up campaign for children missing neonatal vaccination due to shortfalls, as BCG is not recommended for children older than 12 months [15].

A meta-analysis of randomised controlled trials assessing the rate ratio (RR) of TB death in BCG-vaccinated versus $\mathrm{BCG}$-unvaccinated neonates provided a pooled random-effects estimate of 0.34 (95\% confidence interval (CI), 0.12-0.92) [16]. Vaccine efficacy (VE) against TB death is therefore calculated from this rate ratio $(\mathrm{VE}=1-\mathrm{RR})$ as $66 \%$ (95 \% CI, 8-88 \%). Similar estimates were made by Colditz et al. [17] (VE $65 \%$, 95 \% CI, $12-86 \%)$ in a 1995 meta-analysis, but are superseded by the Abubakar review [16]. The Mangtani et al. [18] review does not explore vaccine impact on mortality, so cannot be used for parameterisation of this model. The Abubakar et al. [16] review estimates protection against TB death in the overall population, as opposed to against death given disease, therefore this was incorporated in the model as the proportion of the vaccinated population with complete protection against TB death.

Impact was explored over a 15-year time horizon, as this is the WHO-defined reporting age for childhood TB [19]. Data indicate that BCG provides at least 10 years of protection, and there is some indication of protection still at or beyond 15 years $[16,20]$. Therefore, it is assumed that those vaccinated are protected for the 15year model time horizon. There is no clear evidence to parameterise vaccine waning during this period, so no waning is assumed.

As BCG is contraindicated in HIV-positive neonates [12], and more than $90 \%$ of paediatric HIV infections have been attributed to mother-to-child transmission [21], it is assumed that the vast majority of HIV-positive paediatric TB deaths are not BCG-preventable. Therefore, we 
assume that only HIV-negative paediatric mortality is potentially vaccine preventable (Table 1) [19]. As has been assumed in other BCG modelling studies [8], the annual paediatric TB mortality ('period mortality') is assumed to be equivalent to the number of deaths in a 1-year birth cohort over the first 15 years of life ('cohort mortality'). It is implicit in this assumption that there would be no change in the risk of infection or mortality rates during the 15-year model time horizon.

\section{Model}

A static mathematical model was developed in Microsoft Excel ${ }^{-}$(2013) to estimate the risk of TB death in the first 15 years of life in unprotected infants, the number of paediatric $\mathrm{TB}$ deaths in the first 15 years of life avoided by usual levels of BCG coverage, and the potential number of additional deaths due to 1 year of BCG shortfalls of between $6.3 \%$ and $27.6 \%$ of current global demand.

To estimate the individual-level risk of TB death in the first 15 years of life for children not protected by the vaccine, the number of unprotected children $(n)$ in the birth cohort $(B)$ was first estimated. Those not protected include unvaccinated children and vaccinated children with insufficient immune response for protection.

$$
n=B \times(1-C o v)+B \times C o v \times(1-V E)
$$

The risk of TB death in the first 15 years of life for unprotected children $\left(u_{T B}\right)$ was then calculated by dividing the baseline number of TB deaths (Mort) by the number of unprotected children:

$$
u_{T B}=\frac{M o r t}{n}
$$

For each of the shortfall scenarios $(s=6.3 \%, 10 \%$, $20 \%, 27.6 \%, 100 \%)$, the number of available doses $\left(d_{s}\right)$ and vaccine wastage $(w)$ were employed to calculate vaccine coverage $\left(\mathrm{Cov}_{s}\right)$ of the birth cohort:

$$
\operatorname{Cov}_{s}=\frac{d_{s} \times(1-w)}{B}
$$

The number of children unprotected $\left(n_{s}\right)$ was calculated for each scenario using $\operatorname{Cov}_{s}$ in equation 1. These were used to estimate the number of additional deaths $\left(A_{s}\right)$ for each scenario expected in one birth cohort in the first 15 years of life:

$$
A_{s}=\left(u_{T B} \times n_{s}\right)-\text { Mort }
$$

\section{Uncertainty analysis}

Uncertainty was estimated by sampling values of paediatric $\mathrm{TB}$ mortality and the rate ratio of TB death in vaccinated versus unvaccinated neonates (used to calculate vaccine efficacy) $[16,19]$. The underlying distribution of the TB mortality estimates is not published; therefore, a lognormal distribution was generated from the point estimate $(80,000)$ and $95 \%$ range $(64,000-97,000)$ [19]. A lognormal distribution was also employed for the rate ratio, as the published confidence intervals were estimated using the DerSimonian and Laird method [16, 22]. Location and scale parameters for both distributions were estimated using the $2.5 \%, 50 \%$ and $97.5 \%$ quantiles in the rriskDistributions package in the statistical programme $\mathrm{R}[23,24]$.

A total of 100,000 parameter sets and model outputs were generated according to the prior-defined probabilities using Oracle ${ }^{\circ}$ Crystal Ball (release 11.1.2.4.400, Oracle Corporation, USA). Median and $95 \%$ uncertainty ranges for each estimate were calculated from the 100,000 model outputs.

\section{Results}

It is estimated that one year of global BCG vaccination at usual levels of coverage (90 \%) averts 117,132 (95\% UR: 5049-306,911) TB deaths during the first 15 years of life of that cohort (Table 2). Therefore, without BCG, the point estimate of annual HIV-negative paediatric TB mortality would be estimated to increase from 80,000 to approximately 197,100 . The wide uncertainty ranges derive from the wide confidence intervals around the pooled estimate of the rate ratio of TB death in BCG-vaccinated

Table 2 Estimated additional paediatric TB deaths in BCG supply shortfall scenarios

\begin{tabular}{llllcr}
\hline Supply shortfall (\%) & $\begin{array}{l}\text { Number of doses } \\
\text { shortfall (million) }\end{array}$ & $\begin{array}{l}\text { Global BCG } \\
\text { coverage (\%) }\end{array}$ & $\begin{array}{l}\text { Estimated number of additional paediatric TB deaths } \\
\text { in a 1 year cohort in the first 15 years of life }\end{array}$ & $\begin{array}{l}\text { Increase in number } \\
\text { of deaths (\%) }\end{array}$ \\
\cline { 4 - 5 } & 16.5 & 84 & 7433 & 35 Uncertainty Range & $320-19,477$ \\
10 & 26 & 81 & 11,713 & $505-30,691$ & 14.6 \\
20 & 52 & 72 & 23,426 & $1010-61,382$ & 29.3 \\
27.6 & 71.8 & 65 & 32,347 & $1394-84,755$ & 40.4 \\
100 & 260 & 0 & 117,132 & $5049-306,911$ & 146.4 \\
\hline
\end{tabular}


versus BCG-unvaccinated neonates, as a limited number of studies with relatively small sample sizes from different settings contribute to meta-analyses of this parameter. In our main analysis, it was assumed that the rate ratio of $\mathrm{TB}$ death by vaccination status and the global estimate of paediatric TB deaths were uncorrelated and were thus sampled independently. As some correlation may exist, a post-hoc analysis assessing the sensitivity of the results to correlation assumptions was conducted. Even in the most extreme scenario of $100 \%$ correlation between these two variables, uncertainty ranges around the annual number of TB deaths averted at 2013 vaccination levels narrowed only marginally to $5781-244,556$.

The hypothetical scenarios explored suggest approximately an additional 11,713 (95 \% UR: 505-30,691) paediatric TB deaths in a 1-year cohort in the first 15 years of life per $10 \%$, or 26 million dose, BCG shortfall. This is a $14.6 \%$ increase in the number of deaths.

A 16.5 million dose $(6.3 \%)$ shortfall as reported at the close of 2015, which is reflective of $84 \%$ global coverage, would be estimated to be associated with 7433 (95\% UR: 320-19,477) excess TB deaths in the affected birth cohort in the first 15 years of life (Table 2). A $27.6 \%$ shortfall on global demand was estimated to be associated with 32,347 (95 \% UR: 1394-84,755) additional deaths. Therefore, measures to reduce the shortfall from the anticipated $27.6 \%$ to $6.3 \%$ are estimated to have avoided a possible 24,914 (UR: 1074-65,278) additional deaths.

The modelled estimate of the risk of TB death for an unprotected child in the first 15 years of life $\left(u_{T B}\right)$ was 0.00151 (95 \% UR: 0.00064-0.00299), otherwise expressed as 151 (64-299) per 100,000 children in the first 15 years of life.

\section{Discussion}

Given historical security in BCG supply, the potential public health impact of shortfalls has not previously been quantified. The static mathematical cohort model presented here indicates that even relatively small BCG supply shortfalls could cause sizeable increases in paediatric mortality in the affected cohorts. It is estimated that current coverage of BCG prevents 117,132 (95 \% UR: 5049-306,911) paediatric TB deaths in the first 15 years of life per birth cohort, suggesting that an additional 11,713 (95\% UR: 505-30,691) paediatric deaths could be expected per $10 \%$, or 26 million dose, annual shortfall in BCG supply. Therefore, in the worst case scenario of a 71.8 million dose shortfall anticipated by UNICEF in 2015, up to an additional 32,347 (95\% UR: 1394-84,755) paediatric TB deaths could have been expected in the affected cohort over the 15-year time horizon. However, measures minimising the 2015 shortfall to 16.5 million reduced the estimated number of additional deaths to 7433 (95 \% UR: 320-19,477).

UNICEF procures WHO-prequalified (PQ) BCG from five manufacturers. Adjusting quantities awarded to these manufacturers can meet small-scale changes in demand. However, this supply chain is relatively unresponsive to rapid, or large, changes in supply or demand. In recent years, insufficient supply in the non-UNICEF BCG market has inflated demand for UNICEF-procured PQ vaccine from usually self-procuring countries, such as Egypt, Philippines, Pakistan, and South Africa [6, 25]. Thus, the UNICEF market is vulnerable to challenges faced by any manufacturer, not just those supplying PQ vaccine. During the same period, manufacturing shortfalls were also experienced by two PQ vaccine suppliers $[4,6]$. These concurrent changes in supply and demand for $\mathrm{PQ}$ vaccine far exceeded the responsiveness of the supply system, leading to unmet demand [6].

Rapid and coordinated action from multiple stakeholders to increase supply and to hold buffer stocks at the global rather than national level to allow for prioritisation of limited stocks to high TB-burden countries reduced projected BCG shortages from potentially 71.8 million down to 16.5 million at the end of $2015[5,6]$. The laudable efforts by multiple stakeholders to minimise BCG shortfalls averted an estimated 24,914 (UR: 1074-65,278) additional paediatric deaths. However, due to the remaining shortfall at the close of 2015, it is estimated that a possible 7433 (95\% UR: 320-19,477) additional paediatric TB deaths could be expected in the first 15 years of life of the birth cohort affected in 2015 alone. Therefore, although rapid action in 2015 minimised shortfalls, avoiding a large number of potential additional deaths, the estimated public health impact of even such a relatively small shortfall highlights the importance of ensuring secure manufacturing capacity to meet global BCG demand.

In the estimates presented, the impact of only one year of shortfall was considered, yet recent shortfalls have already extended over several years. Such shortages affecting multiple birth cohorts would result in even greater public health impact. Resolution of the underlying causes takes time; therefore, strategies to pre-emptively avoid shortfalls are essential to avoid unnecessary childhood illness and deaths from TB. New BCG suppliers have been assessed for pre-qualification to increase availability, but future risks include an anticipated $30 \%$ rise in the BCG weighted average price, the sale of one of the UNICEF suppliers making future production plans uncertain, and on-going supply shortages $[5,6]$. It appears unlikely that new manufacturers will enter the market, and there are no known plans for existing suppliers to expand their facilities; therefore, with fixed suppliers and production capacity, mass production of such biologicals will continue to suffer from the small, but very real, risk of production delays.

Reducing vaccine wastage from 20 -dose vials could help maximise utilisation of available vaccines. Our model 
estimates $54.7 \%$ dose wastage, aligning with the $\mathrm{WHO} /$ UNICEF-estimated wastage of approximately $50 \%$ [2]. Wastage is particularly prevalent in smaller health centres, where insufficient neonates present for vaccination during the 6-hour vial use window. Economic modelling has demonstrated that clinics vaccinating fewer than seven infants daily would likely benefit from a 10-dose format [26]. It is also believed that availability of smaller vials would be associated with increased willingness to open vials to provide timely vaccination and increased coverage. Therefore, production of both 10- and 20-dose vials for infant vaccination could allow tailoring of supply by setting, reducing wastage, increasing coverage and potentially reducing the risk of shortages.

Another, longer-term solution to BCG supply issues could be implementation of a more time- and cost-efficient manufacturing process. Current BCG supplies are manufactured by the labour-intensive, classic surface growth method, but a more modern, cost-efficient, dispersed, liquid fermentation process currently in use for novel BCG replacement candidates could potentially provide a more consistent product if used in BCG production and could help ensure adequate global supplies (Personal communication, Ann Ginsberg, Aeras, January 20th 2016).

Mathematical models are, by definition, a simplification of reality. Some specific limitations of this approach are considered. As paediatric cases are generally minimally infectious [27], a static cohort model assuming no transmission nor indirect vaccine effects was employed. This limitation may produce an underestimate of the impact of vaccine shortages on TB mortality. The WHO estimate of HIV-negative childhood TB mortality is a key input in this analysis, yet methodology for estimation of paediatric disease burden is evolving, and therefore the size of the estimates may change in coming years. However, such changes do not affect the proportional increase in the number of deaths expected in each scenario, and therefore the impact of shortfalls could be re-estimated in the future using the proportions reported in Table 2. If countries are contributing cases but are excluded from the denominator due to not having a policy of neonatal BCG vaccination, the model could marginally overestimate the effect of the intervention. However, this is expected to be minimal given paediatric TB is generally a sentinel of high transmission, and in such settings BCG is usually offered.

Uncertainty ranges around the modelled estimates are wide due to large confidence intervals around the vaccine efficacy parameter derived from the Abubakar et al. [16] meta-analysis. Factors contributing to this uncertainty include heterogeneity between study settings and the small number of studies included in the metaanalysis $(n=5)$, with relatively low numbers of person years accrued and very few mortality endpoints. All of the studies were conducted between 1933 and 1960 . New research could help improve the body of evidence for this parameter, and therefore improve modelled estimates of BCG impact. Placebo controlled trials of BCG are no longer ethical due to lack of equipoise, and retrospective observational designs often suffer from substantial biases, but prospective observational studies with larger sample sizes could potentially contribute to the evidence base for this parameter.

It is possible that some HIV-positive TB mortality may be BCG-preventable, as some healthy HIV-positive neonates with unknown HIV status may be vaccinated, and some children become HIV-infected after receipt of BCG. BCG efficacy against mortality is unknown in these groups, but if BCG were to provide partial protection in these children, the reported impact of BCG shortages would be an underestimate.

Although data are limited, it is possible that BCG protection could wane or the duration of protection could be shorter than the modelled period, and therefore the assumption of 15 years of full protection could produce an overestimate of the impact of shortfalls. Conversely, protection is also likely to continue to prevent deaths beyond 15 years of age, but such longer-term impact was outside the scope of this research question.

There is an increasing body of evidence suggestive of a potentially important non-specific beneficial effect of BCG vaccination against all-cause mortality [28]. Controlled trials and observational evidence from the USA, UK and West African settings have reported point estimates of $25-60 \%$ reduction in all-cause mortality, with impact particularly pronounced in the neonatal period $[28,29]$. Therefore, the number of all-cause deaths may also increase with BCG shortfalls, but such non-specific vaccine effects are not accounted for in the model.

It is assumed in the model that neonates missed due to 1-year BCG shortfalls would not receive catch-up vaccination, as WHO does not recommend vaccination above 12 months of age and catch-up campaigns may be difficult to implement [15]. However, for infants under 12 months when supply is restored, catch-up doses may be given to some infants concomitant with other routine Expanded Program on Immunization vaccines, potentially reducing the impact of shortfalls.

UNICEF-reported shortfalls account for demand due to basic country needs and likely some over-procurement to replenish buffer stocks [6]. The scale of over-procurement is unknown and therefore could not be accounted for, but may cause some overestimate of impact in the 2015 scenarios. There are also insufficient data available to quantify the possible mitigating effects of existing buffer stocks in the model. However, in 2014, 28 countries reported BCG stockouts of at least a month at the national level, of which $86 \%$ reported interruption of BCG vaccination 
services [30]; therefore, country reports clearly support the model assumption that global BCG shortages lead to programmatic vaccine shortages.

This global-level model using a single pooled estimate of vaccine efficacy cannot account for likely heterogeneity in the location of stockouts and possible latitudinal variation in vaccine efficacy $[16,18]$. A regional- or countrylevel model would have been desirable, but estimates of country- or regional-level burden of paediatric TB mortality have not yet been published. When supply shortfalls occur, available UNICEF-procured vaccines are prioritised to high TB burden countries [25, 31]. This could not be accounted for in the model due to the lack of the required mortality burden estimates to develop a country-level model, and there are also no data available on how BCG was distributed. Differential distribution of stock could therefore not be accounted for in this global-level model, and thus may overestimate the impact of shortfalls if high burden countries experience fewer shortfalls. However, this mitigation mechanism does not guarantee supply, especially when the global demand gap is large, as demonstrated in 2014 when six of the 22 WHO high-burden countries reported an interruption of vaccination services [30]. This limitation also highlights the importance of UNICEF-led BCG procurement, as left to market forces alone it is likely that many high-burden countries would struggle to procure sufficient vaccine.

\section{Conclusion}

BCG is a mainstay of neonatal vaccination, yet the risks of supply shortages have not previously been explored. It is clear that even relatively small shortfalls have the potential to cause a considerable number of additional paediatric $\mathrm{TB}$ deaths, and the public health risks of larger shortfalls are immense. This is a current issue of global relevance; therefore, continued mobilization to eliminate current shortfalls and development of a secure and responsive mechanism of supply to adequately meet future global demand should be a global public health priority.

\footnotetext{
Abbreviations

BCG: Bacillus Calmette-Guérin; Cl: Confidence intervals; PQ: Pre-qualified; RR: Rate ratio; TB: Tuberculosis; UNICEF: United Nations International Children's Emergency Fund; UR: Uncertainty range; VE: Vaccine efficacy; WHO: World Health Organization
}

\section{Acknowledgments}

We thank Willem Hanekom, Anne Kasmar and Tina Lorenson of the Bill and Melinda Gates Foundation for guidance in the development of this work, and Tom Sumner of the London School of Hygiene and Tropical Medicine for contributions to estimating model uncertainty ranges. We would also like to thank Ann Ginsberg from Aeras, and Heather Deehan, Dorcas Noertoft, Aadrian Sullivan and Ann Ottosen from UNICEF, for providing invaluable feedback on the manuscript.

\section{Funding}

No funding was received specifically for the conduct of this research. $\mathrm{RCH}$ is funded by the UK Medical Research Council (MRC). RGW is funded by the UK Medical Research Council (MRC) and the UK Department for International Development (DFID) under the MRC/DFID Concordat agreement that is also part of the EDCTP2 programme supported by the European Union (MR/J005088/1), the Bill and Melinda Gates Foundation (TB Modelling and Analysis Consortium: OPP1084276, and RSA Evidence for Policy: \#OPP1110334), and UNITAID (4214-LSHTM-Sept15; PO \#8477-0-600). PJD is funded by the STEP TB UNITAID grant to The Global Alliance for TB Drug Development.

\section{Availability of data and materials}

The data supporting the conclusions of this article are included within the article in Table 1.

\section{Authors' contributions}

$\mathrm{RCH}$ and RGW developed the research question and methodology. RCH conducted literature searches, acquired parameter data, developed the mathematical model, and wrote the first manuscript draft. All authors contributed to design and interpretation of the model and writing of the manuscript. All authors approved the final manuscript.

\section{Competing interests}

$\mathrm{RCH}$ has provided consultancy for GSK Vaccines outside the submitted work and unrelated to this research topic. All other authors report no potential competing interests.

\section{Consent for publication}

Not applicable.

\section{Ethics approval and consent to participate} Not applicable.

\section{Author details}

${ }^{1}$ TB Modelling Group, TB Centre and Centre for the Mathematical Modelling of Infectious Diseases, Faculty of Epidemiology and Population Health, London School of Hygiene \& Tropical Medicine, Keppel Street, London WC1E 7HT, UK. ${ }^{2}$ School of Health and Related Research, University of Sheffield, 30 Regent Street, Sheffield S1 4DA, UK.

Received: 24 May 2016 Accepted: 2 September 2016

Published online: 15 September 2016

\section{References}

1. World Health Organization. Reported Estimates of BCG Coverage. http://apps.who.int/immunization_monitoring/globalsummary/timeseries/ tscoveragebcg.html. Accessed 23 Dec 2015.

2. World Health Organization. Immunization Financing Indicators from the WHO-UNICEF Joint Reporting Form. Geneva: WHO; 2014.

3. World Health Organization. Immunization Standards: BCG (10/20 dose vial). http://www.who.int/immunization_standards/vaccine_quality/118_bcg/en/. Accessed 30 Dec 2015

4. United Nations Children's Emergency Fund. BCG Vaccine: Current Supply \& Demand Outlook (September 2013). http://www.unicef.org/supply/files/ BCG_Supply_Status_Sept_2013.pdf. Accessed 15 June 2015.

5. United Nations Children's Emergency Fund. BCG Vaccine: Current Supply \& Demand Outlook (December 2014). http://www.unicef.org/supply/files/ BCG_Supply_Status_December_2014.pdf. Accessed 15 June 2015.

6. United Nations Children's Emergency Fund. BCG Vaccine Supply \& Demand Outlook (December 2015). http://www.unicef.org/supply/files/BCG_Supply_ Status_December_2015.pdf. Accessed 15 June 2015.

7. Trunz BB, Fine P, Dye C. Effect of BCG vaccination on childhood tuberculous meningitis and miliary tuberculosis worldwide: a meta-analysis and assessment of cost-effectiveness. Lancet. 2006;367(9517):1173-80.

8. Rahman M, Sekimoto M, Takamatsu I, Hira K, Shimbo T, Toyoshima K, Fukui T. Economic evaluation of universal BCG vaccination of Japanese infants. Int J Epidemiol. 2001;30(2):380-5.

9. United Nations Children's Emergency Fund. BCG Vaccine: Current Supply \& Demand Outlook (August 2014). http://www.unicef.org/supply/files/BCG Supply_Status_August_2014.pdf. Accessed 27 July 2016. 
10. Zwerling A, Behr M, Verma A, Brewer T, Menzies D, Pai M. The BCG Global Atlas. http://www.bcgatlas.org. Accessed 23 Dec 2015.

11. United Nations Population Division. World Population Prospects, the 2015 Revision. http://esa.un.org/unpd/wpp/Download/Standard/Population/. Accessed 21 Dec 2015.

12. World Health Organization. Global Advisory Committee on Vaccine Safety, 29-30 November 2006. Wkly Epidemiol Rec. 2007;82(3):18-24.

13. United Nations Programme on HIV/AIDS. UNAIDS Report on the Global AIDS Epidemic 2013. http://www.unaids.org/sites/default/files/en/media/ unaids/contentassets/documents/epidemiology/2013/gr2013/UNAIDS_ Global_Report 2013 en.pdf. Accessed 18 June 2015.

14. Harris JB, Gacic-Dobo M, Eggers R, Brown DW, Sodha SV. Global routine vaccination coverage, 2013. MMWR Morb Mortal Wkly Rep. 2014;63(46): $1055-8$.

15. World Health Organization. Recommendations for Interrupted or Delayed Routine Immunization - Summary of WHO Position Papers. http://www. who.int/immunization/policy/Immunization_routine_table3.pdf?ua=1. Accessed 7 Jan 2016

16. Abubakar I, Pimpin L, Ariti C, Beynon R, Mangtani P, Sterne JA, Fine PE, Smith PG, Lipman M, Elliman D, et al. Systematic review and meta-analysis of the current evidence on the duration of protection by Bacillus CalmetteGuerin vaccination against tuberculosis. Health Technol Assess. 2013;17(37): 1-372. v-vi.

17. Colditz GA, Berkey CS, Mosteller F, Brewer TF, Wilson ME, Burdick E, Fineberg HV. The efficacy of Bacillus Calmette-Guerin vaccination of newborns and infants in the prevention of tuberculosis: meta-analyses of the published literature. Pediatrics. 1995;96(1 Pt 1):29-35.

18. Mangtani P, Abubakar I, Ariti C, Beynon R, Pimpin L, Fine PEM, Rodrigues LC, Smith PG, Lipman M, Whiting PF, et al. Protection by BCG against tuberculosis: a systematic review of randomised controlled trials. Clin Infect Dis. 2014;58(4):470-80.

19. World Health Organization. Global Tuberculosis Report 2014. http://apps, who.int/iris/bitstream/10665/137094/1/9789241564809_eng.pdf. Accessed 22 Jan 2016.

20. Nguipdop-Djomo P, Heldal E, Rodrigues LC, Abubakar I, Mangtani P. Duration of BCG protection against tuberculosis and change in effectiveness with time since vaccination in Norway: a retrospective population-based cohort study. Lancet Infect Dis. 2016;16(2):219-26.

21. World Health Organization. Care of the HIV-exposed or infected newborn. http://www.who.int/maternal_child_adolescent/topics/newborn/care_of_ hiv_exposed/en/. Accessed 22 Jan 2016.

22. DerSimonian R, Laird N. Meta-analysis in clinical trials. Control Clin Trials. 1986;7(3):177-88.

23. Belgorodski K, Greiner M, Tolksdorf K, Schueller K. rriskDistributions: Fitting Distributions to Given Data or Known Quantiles. R package version 2.1. 2015.

24. R Core Team. R: A Language and Environment for Statistical Computing. Vienna: R Foundation for Statistical Computing; 2015.

25. United Nations Children's Emergency Fund. UNICEF Information to Countries on Constrained BCG Vaccine Market. http://www.unicef.org/ supply/index_82351.html. Accessed 21 Dec 2015.

26. Lee BY, Norman BA, Assi T-M, Chen S-I, Bailey RR, Rajgopal J, Brown ST, Wiringa AE, Burke DS. Single versus multi-dose vaccine vials: an economic computational model. Vaccine. 2010;28(32):5292-300

27. Starke JR. Transmission of mycobacterium tuberculosis to and from children and adolescents. Semin Pediatr Infect Dis. 2001;12(2):115-23.

28. Aaby P, Roth A, Ravn H, Napirna BM, Rodrigues A, Lisse IM, Stensballe L, Diness BR, Lausch KR, Lund N, et al. Randomized trial of BCG vaccination at birth to low-birth-weight children: beneficial nonspecific effects in the neonatal period? J Infect Dis. 2011;204(2):245-52.

29. Garly ML, Martins CL, Bale C, Balde MA, Hedegaard KL, Gustafson P, Lisse IM, Whittle HC, Aaby P. BCG scar and positive tuberculin reaction associated with reduced child mortality in West Africa. A non-specific beneficial effect of BCG? Vaccine. 2003;21(21-22):2782-90.

30. World Health Organization. WHO vaccine-preventable diseases: monitoring system. 2015 Global Summary. http://apps.who.int/immunization monitoring/globalsummary/indicators. Accessed 23 Dec 2015.

31. World Health Organization. Guidance on How to Prioritize Globally Constrained BCG Vaccine Supply to Countries. http://www.who.int/immunization/diseases/ tuberculosis/BCG-country-prioritization.pdf. Accessed 5 Jan 2016.

\section{Submit your next manuscript to BioMed Central and we will help you at every step:}

- We accept pre-submission inquiries

- Our selector tool helps you to find the most relevant journal

- We provide round the clock customer support

- Convenient online submission

- Thorough peer review

- Inclusion in PubMed and all major indexing services

- Maximum visibility for your research

Submit your manuscript at www.biomedcentral.com/submit
O) Biomed Central 